\title{
Improvement of the management of anticoagulation in Atrial Fibrillation patients in the emergency and acute medical departments; a service quality improvement report
}

\author{
Amr Elkammash $^{1}$; Yosra Taha ${ }^{1} ;$ Saleh Kanaan ${ }^{1}$; Martin Taylor ${ }^{1}$ \\ 1: Department of Acute Medicine, University Hospitals Dorset, The Royal Bournemouth Hospital, \\ Bournemouth, UK. \\ Corresponding author: Amr Elkammash. Email: amr.elkammash@nhs.net
}

ORCHID ID: https://orcid.org/0000-0002-9322-3347

\begin{abstract}
Atrial fibrillation (AF) is considered one of the main causes of ischemic stroke. The $\mathrm{CHA}_{2} \mathrm{DS}_{2} \mathrm{VASC}$ score can predict the stroke risk. Proper anticoagulation can significantly reduce such risk. Anticoagulation involves a risk of bleeding, which can be predicted by the HAS-BLED score. The non-documentation of both AF risk scores in the medical notes of patients presenting with acute or paroxysmal AF has alerted our team, for fear of missing administering the proper anticoagulation. A baseline audit showed that the $\mathrm{CHA}_{2} \mathrm{DS}_{2}$ VASC score was documented in $27 \%$ of such patients, while the HAS-BLED score was documented in $5 \%$ of them. A quality improvement project was planned and included two PDSA cycles over a period of five months. The first cycle was based on raising the awareness of the junior doctors on risk assessment of AF patients and the importance of anticoagulation prescription. This involved educational posters, emails, and presentations. On the second cycle, the baseline audit showed a $69 \%$ improvement (from $27 \%$ to $46 \%$ ) in the rate of $\mathrm{CHA}_{2} \mathrm{DS}_{2} \mathrm{VASC}$ score assessment and a $140 \%$ improvement (from $5 \%$ to $12 \%$ ) in the rate of HAS-BLED scoring. However, the rate of anticoagulation prescription remained almost the same in both cycles (55\% and 52\%). A doctors' survey showed that they are not quite comfortable prescribing long-term anticoagulation on acute care units because of the inadequate assessment of the bleeding risk, and the associated comorbidities that can disturb the action of the oral anticoagulants. They preferred to refer the patient to a specialised AF clinic for further assessment and prescription. On auditing the patient referral on discharge, it was found that $91 \%$ of the patient were seen at the AF clinic in 2 weeks. The remainders were referred to their GP for further assessment and prescription. That seemed to be a safer way of anticoagulation prescription for such patients.
\end{abstract}

Keywords: Atrial fibrillation; anticoagulation; quality improvement; emergency; acute. 


\section{Introduction:}

Atrial fibrillation (AF) forms a huge health burden on the healthcare system in the UK and worldwide. In the UK, it represents $6 \%$ of all acute hospital admissions and $33 \%$ of admissions for cardiac arrhythmias. It is responsible for different thromboembolic complications including ischemic stroke. ${ }^{1}$ Ischemic stroke is a major cause of cardiovascular mortality. One-third of ischemic strokes are estimated to be caused by $\mathrm{AF}^{2}$ The risk of stroke can be effectively predicted by calculating the $\mathrm{CHA}_{2} \mathrm{DS}_{2}$ VASC score. ${ }^{3}$ Such risk can be effectively reduced by administration of the suitable anticoagulation; orally or parenterally. The savings due to adverse event reduction in AF patients after oral anticoagulation prescription in the UK are estimated to be 13.5 million pounds. ${ }^{4}$ Anticoagulation prescription carries a risk of bleeding. Such risk can be predicted using the HAS-BLED score. ${ }^{5}$ The European Society of Cardiology (ESC) and the National Institute for Health and Clinical Excellence (NICE) recommended the calculation of $\mathrm{CHA}_{2} \mathrm{DS}_{2}$ VASC and HAS-BLED for all AF patients to guide the decision regarding the anticoagulation prescription. ${ }^{6,7}$ Acute care units, including the Emergency department (ED) and Acute Medical Unit (AMU), have special challenges that may hinder anticoagulation prescription to AF patients. These include time constraints and clinically unwell patients. ${ }^{8}$

We identified low use of $\mathrm{CHA}_{2} \mathrm{DS}_{2} \mathrm{VASC}$ and HAS-BLED scores in patients presenting with AF ( acute or paroxysmal). This might reflect inadequate prescription of oral anticoagulants to indicated patients. Therefore, we designed a quality improvement project aiming at the improvement of the practice of AF patients' assessment and prescription of oral anticoagulants in the acute care units.

\section{Methods:}

The working team included three senior house officer doctors, under the supervision of a consultant physician in Acute Medicine. Initially, we audited AF risk scoring and anticoagulation prescription. The audit included 60 patients presenting to the acute care units with either a new or a paroxysm of non sustained AF. It was done retrospectively between March 2019 and March 2020 using electronic records. We collected the data using an online electronic proforma. The trust's clinical audit team carried out the data analysis. The proposed minimum accepted compliance rate for both $\mathrm{CHA}_{2} \mathrm{DS}_{2}$ VASC and HAS-BLED scores was $90 \%$, and that for anticoagulation prescription was $95 \%$. In this audit, the $\mathrm{CHA}_{2} \mathrm{DS}_{2}$ VASC and HAS-BLED scores were calculated in $27 \%$ and $5 \%$ of the patients respectively. An anticoagulant was prescribed in 55\% of the patients. (Fig 1) The AMU performed slightly better than $\mathrm{ED}$ regarding $\mathrm{CHA}_{2} \mathrm{DS}_{2} \mathrm{VASC}$ score calculation and anticoagulation prescription. (Fig 2)

\section{The first cycle of PDSA:}

The team suggested that the causes of the gap between the actual performance and the minimum compliance rate may include the inability of doing the risk scores within the high workloads; the underdocumentation of the risk scores; and the unawareness of the national guidelines and the local policy. Therefore, we put an action plan to target these causes. We designed an awareness-raising campaign to educate junior doctors on the magnitude of the problem and the national recommendations on the management of AF patients. The campaign involved the presentation of eye-catching posters among the acute care clinical areas including the easy-memorable Acronym SBAN ( Stroke risk; Bleeding risk; Anticoagulate). We also ran our posters as screen savers on the computers across the hospital. Educational emails were sent to all junior doctors including the NICE guidance and local policy recommendations. The team presented the audit results in different scientific meetings including the Medical Grand Round, the Cardiology department MDT meeting and the trust's clinical governance meeting. We planned a follow-up audit in a five-month time, taking into consideration the COVID pandemic situation. This cycle ran between December 2020 and April 2021.

The second cycle of PDSA: 
This cycle started with an audit that included 33 patients who presented to the acute care units with either a new or a paroxysm of AF between December 2020 and April 2021 (the same period of implementation of the improvement plan). We studied those patients retrospectively using their electronic records. We used an electronic online proforma to collect data, and these were analysed by the clinical audit team. This audit showed a $69 \%$ improvement in the rate of $\mathrm{CHA}_{2} \mathrm{DS}_{2} \mathrm{VASC}$ score assessment (from $27 \%$ to $46 \%$ ) and a $140 \%$ improvement in HAS-BLED score assessment (from 5\% to $12 \%$ ). However, the rate of anticoagulation prescription remained almost the same (55\% and 52\% in the first and second cycles respectively). ( Fig 3)

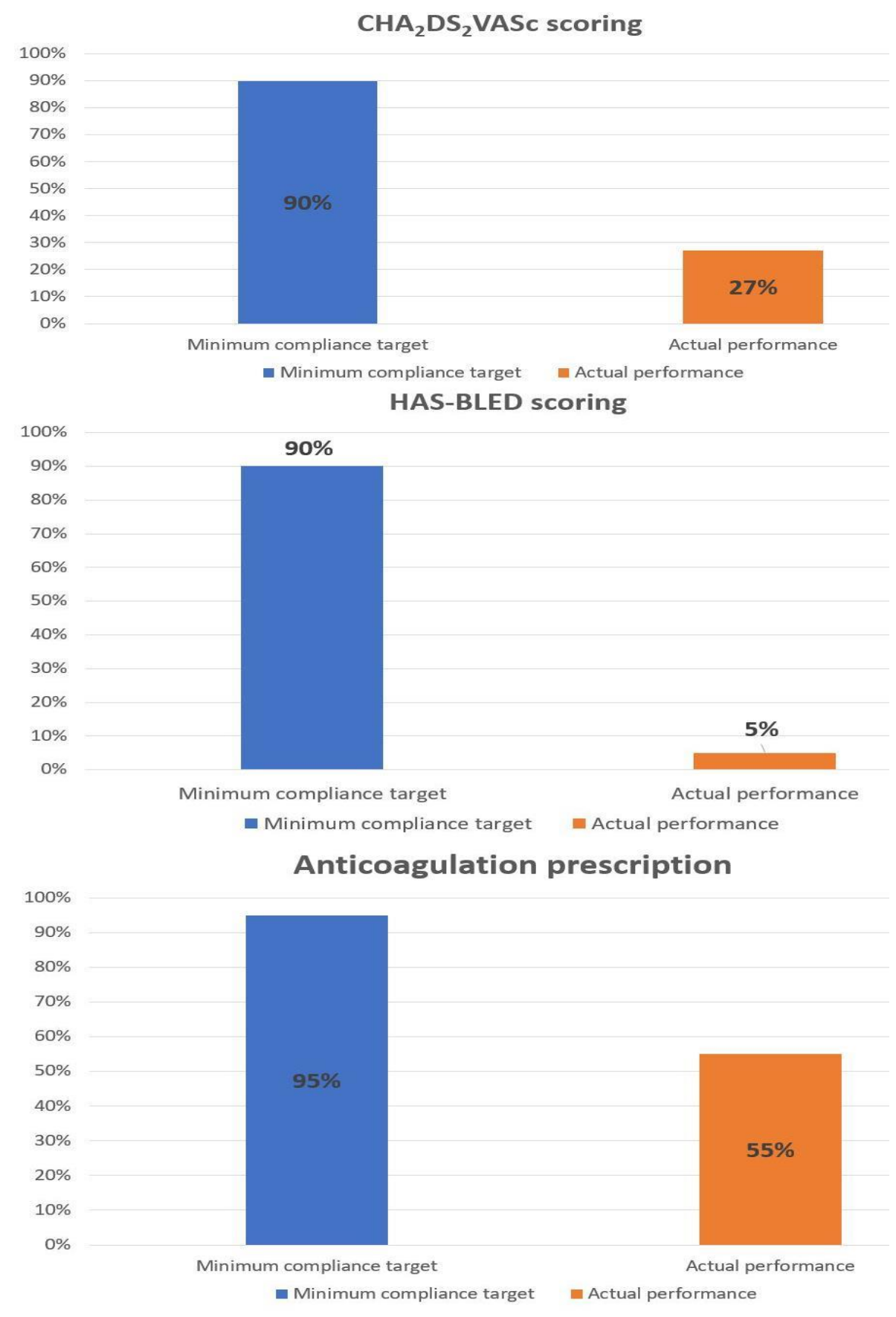

Fig 1: The performance across the acute care units regarding AF risk scoring and anticoagulation presciption 


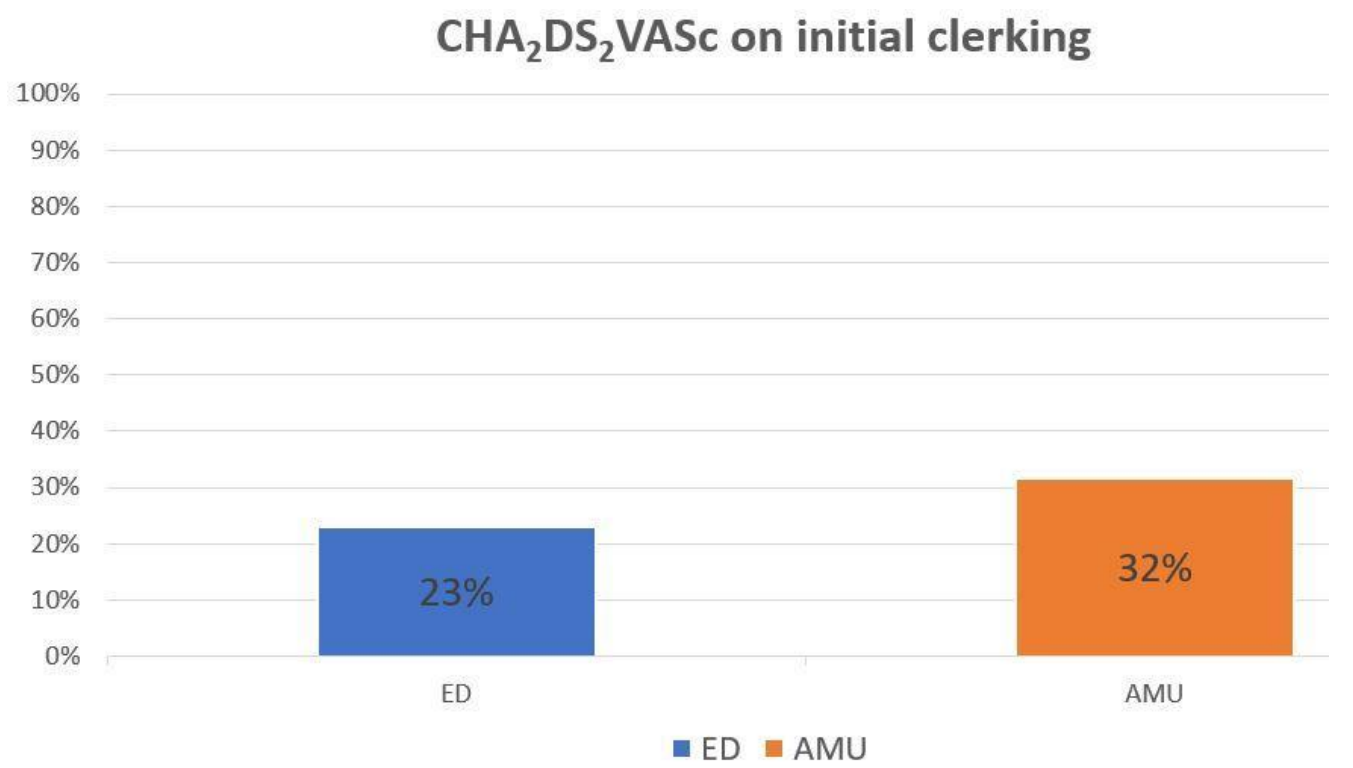

Anticoagulation is given

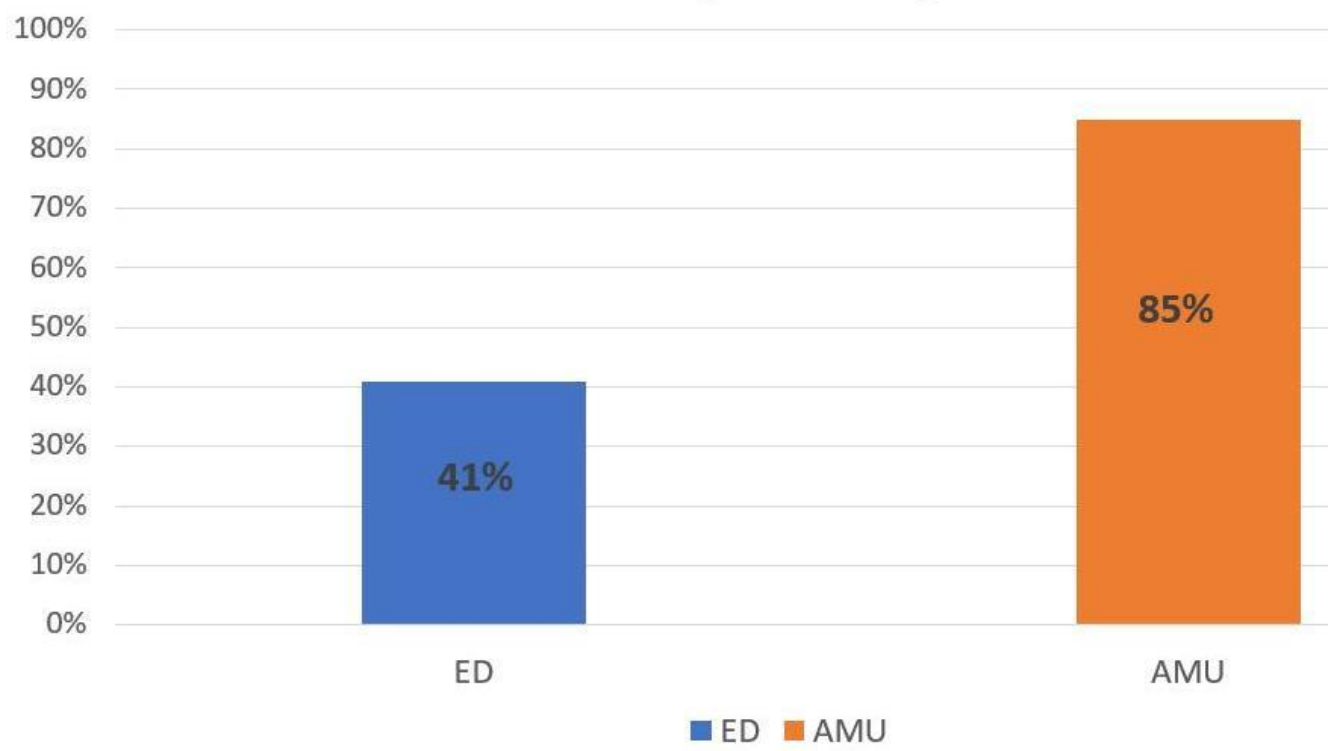

Fig 2: Difference between ED and $A M U$ in $\mathrm{CHA}_{2} \mathrm{DS}_{2} \mathrm{VASC}$ score calculation and anticoagulation prescription 


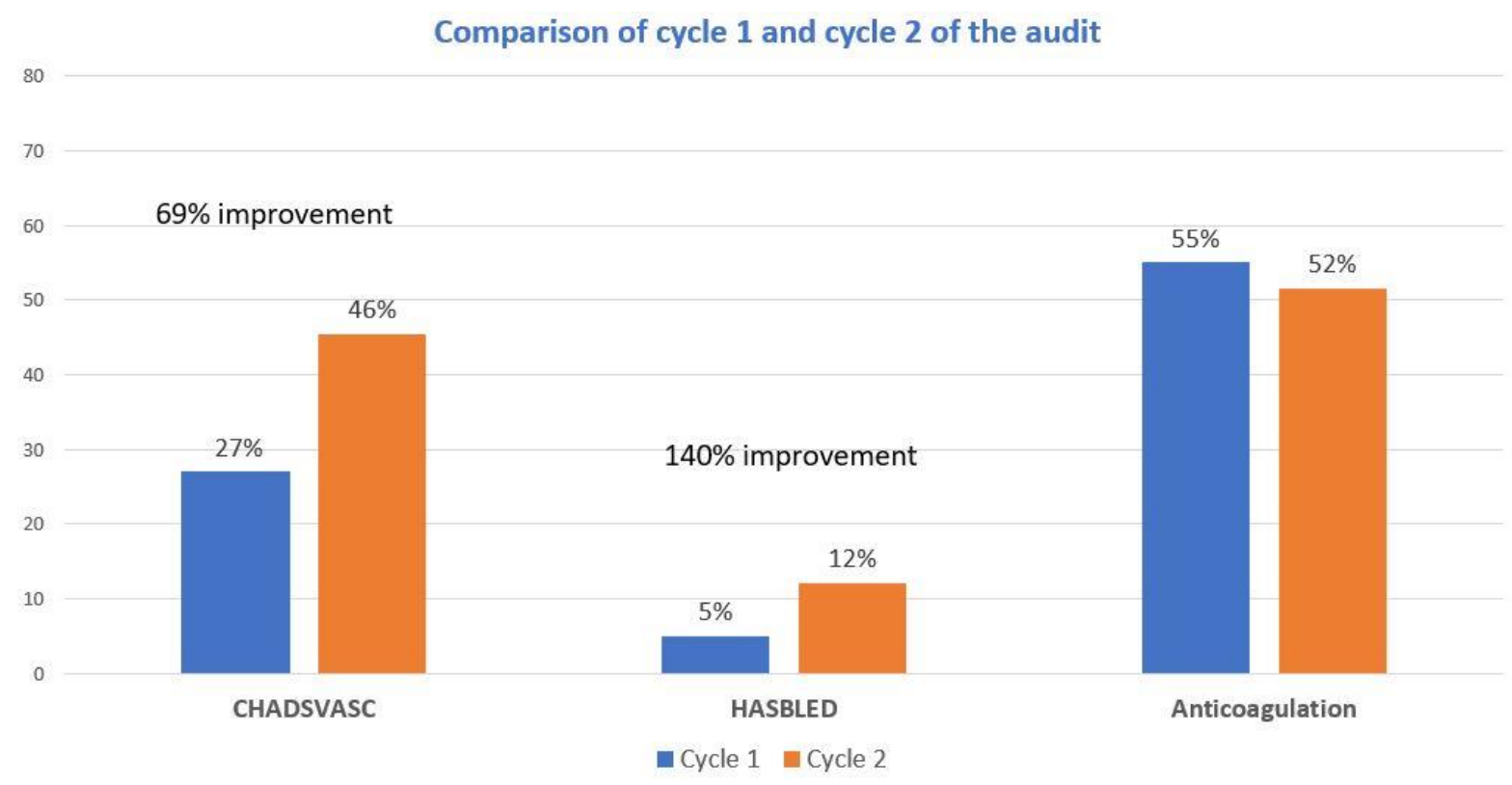

Fig 3: Comparison of the AF risk scoring and anticoagulation prescription between the first and second cycles.

Despite the noticeable improvement in risk scores assessment, anticoagulation prescription did not change across both cycles. Our team surveyed the junior doctors working in the acute care sites and found that the majority of them (93\%) did not feel comfortable prescribing long-term anticoagulation in an acute setting. In such a setting, there is a time constraint on an in-depth discussion regarding the importance of anticoagulation and assessment of the bleeding risk. Acute medical patients may also have disturbances of the kidney and/or liver function, which can affect the kinetics of action of the oral anticoagulants. Doctors preferred to refer the patients to the AF clinic, where patients present in a stable clinical condition and have more time for anticoagulation discussions.

Based on our findings, we re-audited the same 33 patients included in the second cycle regarding the rate of their referral after their acute admission to a proper healthcare facility for anticoagulation discussion and prescription. We found that $91.4 \%$ of the patients were referred to AF clinic on discharge, and those were seen in a two-week time. The time to assessment was discussed with a consultant cardiologist and electrophysiologist and was found safe for those patients. The remaining patients were referred back to their GP for anticoagulation assessment and prescription. We felt that patients' safety was not undermined by anticoagulation prescription as an outpatient.

\section{Discussion:}

This project aimed at improving the management of anticoagulation in AF patients in the acute setting. It tried to ingrain the culture of the use of $\mathrm{CHA}_{2} \mathrm{DS}_{2} \mathrm{VASC}$ and HAS-BLED risk scores to guide anticoagulation prescription. The project managed to increase the use of these scores and highlighted the challenges facing anticoagulation prescription in the acute setting.

Oral anticoagulants are the most frequently incriminated medication in fatal drug errors and legal claims. ${ }^{9}$ Lack of the proper skills and environment for bleeding risk assessment can lead to unsafe prescription. Patient factors such as memory problems can also make the task more difficult and can lead to lethal consequences if health professionals do not give the appropriate time for counselling. ${ }^{10}$ Acute care environments are quite busy with a lot of disruptions that can cause documentation and communication errors. ${ }^{11}$ All these factors caused a reduction in the rate of anticoagulation prescription 
which was corroborated in this project. However, a timely referral to a specialised facility (e.g. AF clinic) seems to be a safe solution for the anticoagulation prescription to acute patients.

A limitation to this project is that it was run at the time of the COVID pandemic, which added a lot of pressure on the health care facilities. This may have lead to a reduced rate of risk score calculation and anticoagulant prescription to acute AF patients. However, patient safety was not endangered as they were referred appropriately to the AF clinic. The pandemic also limited our chances to give face-toface education to the medical staff on the topic.

In conclusion, our project managed to raise the awareness of junior doctors to the importance of usage of $\mathrm{CHA}_{2} \mathrm{DS}_{2} \mathrm{VASC}$ and HAS-BLED scores on assessment of AF patients. It also confirmed the maintenance of a safe anticoagulant prescription in the acute setting of ED or AMU. Potential for further improvement in the care of AF patients can be through the use of the $\mathrm{CHA}_{2} \mathrm{DS}_{2} \mathrm{VASC}$ score as a triaging tool to decide the urgency of patient assessment by GP or AF clinic.

\section{References:}

1. GY L, HF T. Management of atrial fibrillation. Lancet (London, England) 2007; 370: 604618.

2. Cowan JC, $\mathrm{Wu}$ J, Hall M, et al. A 10 year study of hospitalized atrial fibrillation-related stroke in England and its association with uptake of oral anticoagulation. Eur Heart J 2018; 39: 2975-2983.

3. GY L, R N, R P, et al. Refining clinical risk stratification for predicting stroke and thromboembolism in atrial fibrillation using a novel risk factor-based approach: the euro heart survey on atrial fibrillation. Chest 2010; 137: 263-272.

4. NATIONAL INSTITUTE FOR HEALTH AND CARE EXCELLENCE QUALITY AND OUTCOMES FRAMEWORK (QOF) INDICATOR DEVELOPMENT PROGRAMME Cost impact statement: Atrial Fibrillation Proposed change to QOF indicator: NNM45/AF004 and NNM46 / AF005. 2014.

5. R P, DA L, R N, et al. A novel user-friendly score (HAS-BLED) to assess 1-year risk of major bleeding in patients with atrial fibrillation: the Euro Heart Survey. Chest 2010; 138: 10931100.

6. Recommendations | Atrial fibrillation: diagnosis and management | Guidance | NICE, https://www.nice.org.uk/guidance/ng196/chapter/Recommendations (accessed 11 July 2021).

7. Hindricks G, Potpara T, Dagres N, et al. 2020 ESC Guidelines for the diagnosis and management of atrial fibrillation developed in collaboration with the European Association for Cardio-Thoracic Surgery (EACTS)The Task Force for the diagnosis and management of atrial fibrillation of the European Society of Cardiology (ESC) Developed with the special contribution of the European Heart Rhythm Association (EHRA) of the ESC. Eur Heart J 2021; 42: 373-498.

8. Kremers MNT, Nanayakkara PWB, Levi M, et al. Strengths and weaknesses of the acute care systems in the United Kingdom and the Netherlands: what can we learn from each other? BMC Emerg Med 2019191 2019; 19: 1-7. 
9. T D, R G. Anticoagulation service: improving the referral process. BMJ Qual Improv reports 2014; 3: u204229.w1833.

10. Keers RN, Williams SD, Cooke J, et al. Causes of Medication Administration Errors in Hospitals: a Systematic Review of Quantitative and Qualitative Evidence. Drug Saf 2013; 36: 1045.

11. Yu KT, Green RA. Critical Aspects of Emergency Department Documentation and Communication. Emerg Med Clin North Am 2009; 27: 641-654.

Conflicts of interests: nothing to declare.

Acknowledgement: The team would like to thank Dr Richard Balasubramaniam (Consultant Cardiologist) and Dr Peter Swallow ( Consultant Emergency physician) for their support of this project. 